\title{
A General Model of Zoonosis Where the Diseases Can Only Be Transmitted from Animals to Humans
}

\author{
Xueying Liu \\ Department of Mathematics, Shanghai University, Shanghai, China \\ Email: dittol@i.shu.edu.cn
}

How to cite this paper: Liu, X.Y. (2019) A General Model of Zoonosis Where the Diseases Can Only Be Transmitted from Animals to Humans. Advances in Pure Mathematics, 9, 67-77.

https://doi.org/10.4236/apm.2019.92005

Received: January 21, 2019

Accepted: February 16, 2019

Published: February 19, 2019

Copyright $\odot 2019$ by author(s) and Scientific Research Publishing Inc. This work is licensed under the Creative Commons Attribution International License (CC BY 4.0).

http://creativecommons.org/licenses/by/4.0/

\section{cc) (i) Open Access}

\begin{abstract}
Zoonosis is an important factor affecting human economic development and population mortality. This paper introduces a general model of zoonosis, in which the diseases can only be transmitted from animals to humans, such as rabies, brucellosis and so on. The basic reproduction number $R_{0}$ is derived. And then the global stability of the disease-free equilibrium and endemic equilibrium models is analyzed by using the method of comparison principle and Lyapunov function. Next, a numerical analysis is performed to elaborate the consistency of theoretical and numerical results and to prove the practical significance of zoonosis research. The numerical results show that our models are applicable to zoonosis with animal size larger than or smaller than population size. Finally, in order to see the most important factor for the epidemic of zoonosis a sensitive analysis is analyzed.
\end{abstract}

\section{Keywords}

Zoonosis, Global Stability, Equilibrium, Sensitive Analysis

\section{Introduction}

Infectious diseases seriously endanger human health and the development of community economy all over the world [1] [2] [3], historically, infectious diseases have caused millions of deaths. SARS attracted worldwide attention in 2003, more than 8000 infected people have been found worldwide, resulting in more than 900 deaths [4]; Dengue fever in Thailand has reached 5284 people, and the disease is prevalent in Ecuador, Paraguay, Australia and other countries to varying degrees by 2006 [5]. Thus, researching and controlling the dynamic process of infections is very significant to society. 
Among numerous infectious diseases, zoonosis is an important factor affecting human economic development and population mortality. There are about 200 confirmed zoonoses in the world, such as avian influenza, rabies, malaria, brucellosis and so on. From 1996 to 2015, 30,300 human rabies cases were reported [6]. Every year, about 50,000 people are newly infected with brucellosis [7]. What's more, there are about 300 to 500 million new cases because of malaria annual, among which maybe one million to three million deaths, most of them are children [8]. Malaria kills a child every 40 seconds, costing the world more than 2000 young lives a day [8] [9].

Many scholars have studied infectious disease models. At the beginning Kermack and Mckendrick opened the door of infectious disease research. In 1927 and 1932, they separately established the classical SIR [10] and SIS [11] model of plague. In 2002 van Driessche and Watmough [12] provided a method of establishing Lyapunov function which furnishes strong theoretical support for the latter study of the dynamic process of the infections. On this basis, more and more scholars established different models for different infectious diseases [13] [14] [15]. However, a basic model on zoonosis has not been established at now. Many models consider the vaccination [12] [16], but there are only a few people get vaccinated in poor area, even there is not an effective vaccination for some newly discovered zoonoses. Some models consider human transmission to human or animal [17] [18], in fact there are some zoonosis that can only be transmitted from animals to humans such as rabies, brucellosis, tapeworm disease and so on. What's more, few animals are treated after they get sick in the real life. This paper studies on this kind of zoonosis.

In this article, a general model of the interaction between infected animals and humans are established. Referencing to classical model [10] [11] we generalize a SIS model which consider the cure rate those who are infected on human population and a $S I$ model on animal population. The organizational structure of the article is shown below. In Section 2 a mathematical expression of the model is constructed. The third section studies the global dynamics of disease from the perspective of disease-free equilibrium and endemic equilibrium. Section 4 makes numerical simulation to explain the consistency of the theory and numerical results, after that the sensitive analysis is performed. Finally, the conclusion is shown in Section 5 .

\section{Model Formulation}

We consider both animal and human, then classify each of them into tow subclasses: susceptible and infectious. Defining $S_{1}, I_{1}$ as the susceptible and infectious human, $S_{2}, I_{2}$ as the susceptible and infectious animals respectively. If a susceptible human individual is contact with infected animals, this human individual may be infected. If this one received effective treatment, he can change back to a susceptible individual, but if he isn't cure in time, he is in the risk of death from infection. The model proposed in this paper is a system composed of four ordinary differential equations: 


$$
\begin{aligned}
& \frac{\mathrm{d} S_{1}}{\mathrm{~d} t}=b_{1}+\sigma_{1} I_{1}-\beta_{1} S_{1} I_{2}-d_{1} S_{1} \\
& \frac{\mathrm{d} I_{1}}{\mathrm{~d} t}=\beta_{1} S_{1} I_{2}-\sigma_{1} I_{1}-\alpha_{1} I_{1}-d_{1} I_{1} \\
& \frac{\mathrm{d} S_{2}}{\mathrm{~d} t}=b_{2}-\beta_{2} S_{2} I_{2}-d_{2} S_{2} \\
& \frac{\mathrm{d} I_{2}}{\mathrm{~d} t}=\beta_{2} S_{2} I_{2}-\alpha_{2} I_{2}-d_{2} I_{2}
\end{aligned}
$$

The dynamic transmissions for the model of infectious of animal and humans are demonstrated in Figure 1.

Figure 1 illustrate that the people and animal population import new susceptible individuals by the birth of baby with the number of $b_{1}$ and $b_{2}$. Under the influence of infected animals, the susceptible individuals will transfer to infected one with the rate of $\beta_{1}$ and $\beta_{2}$. In the people population, the infected individual can return back to a susceptible individual with the rate $\sigma_{1}$ by medical treatment. The infected individuals will die and then be removed from the population for the reason of nature factor with probability of $d_{1}, d_{2}$ and disease factor with probability of $\alpha_{1}, \alpha_{2}$. All the parameters with subscript 1 define the people population property while the parameters with subscript 2 define the animal population property. Based on the reality, we can know there is not a parameter is negative for human population.

\section{Dynamic Analysis}

Following, we express the derivative of $t$ with $S_{1}^{\prime}, I_{1}^{\prime}, S_{2}^{\prime}, I_{2}^{\prime}$. Adding the first two equations, we can get

$$
S_{1}^{\prime}+I_{1}^{\prime}=b_{1}-d_{1} S_{1}-d_{1} I_{1}-\alpha_{1} I_{1} \leq b_{1}-d_{1} S_{1}-d_{1} I_{1}
$$

There is

$$
\lim _{t \rightarrow \infty} S_{1}+I_{1}=\frac{b_{1}}{d_{1}}
$$

Similarly

$$
\lim _{t \rightarrow \infty} S_{2}+I_{2}=\frac{b_{2}}{d_{2}}
$$

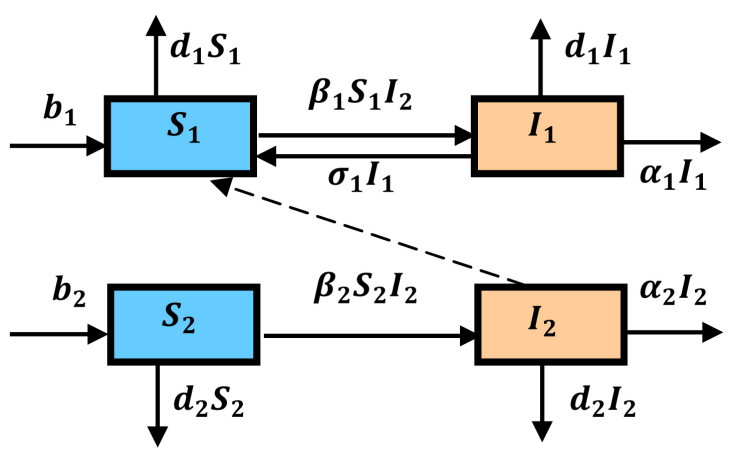

Figure 1. Transmission diagram of zoonosis among animals and humans. 
Accordingly, we can conclude an invariant set for the model

$$
\Omega=\left\{\left(S_{1}, I_{1}, S_{2}, I_{2}\right) \in R_{+}^{4} \mid 0 \leq S_{1}+I_{1} \leq \frac{b_{1}}{d_{1}}, 0 \leq S_{2}+I_{2} \leq \frac{b_{2}}{d_{2}}\right\}
$$

\subsection{The Basic Reproduction Number}

Obviously, setting the right-hand side of the four differential equations to zero we can find the equilibrium. When set $I_{1}=I_{2}=0$ The model (1) has a unique disease-free equilibrium $E^{0}$ :

$$
E^{0}=\left(S_{1}^{0}, I_{1}^{0}, S_{2}^{0}, I_{2}^{0}\right)=\left(\frac{b_{1}}{d_{1}}, 0, \frac{b_{2}}{d_{2}}, 0\right)
$$

when $I_{1}=I_{2} \neq 0$, we can find a unique equilibrium $E^{*}$ :

$$
E^{*}=\left(S_{1}^{*}, I_{1}^{*}, S_{2}^{*}, I_{2}^{*}\right)
$$

where

$$
\begin{array}{ll}
S_{2}^{*}=\frac{\alpha_{2}+d_{2}}{\beta_{2}} & I_{2}^{*}=\frac{b_{2} \beta_{2}-d_{2}\left(\alpha_{2}+d_{2}\right)}{\beta_{2}\left(\alpha_{2}+d_{2}\right)} \\
S_{1}^{*}=\frac{b_{1}\left(\alpha_{1}+d_{1}+\sigma_{1}\right)}{\beta_{1} I_{2}^{*}\left(d_{1}+\alpha_{1}\right)+d_{1}\left(\alpha_{1}+d_{1}+\sigma_{1}\right)} & I_{1}^{*}=\frac{b_{1}-d_{1} S_{1}^{*}}{d_{1}+\alpha_{1}}
\end{array}
$$

Based on this, the knowledge of next generation matrix and basic reproduction number are proposed [10], we now consider the follow auxiliary model

$$
\begin{aligned}
& I_{1}^{\prime}=\beta_{1} S_{1} I_{2}-\sigma_{1} I_{1}-\alpha_{1} I_{1}-d_{1} I_{1} \\
& I_{2}^{\prime}=\beta_{2} S_{2} I_{2}-\alpha_{2} I_{2}-d_{2} I_{2}
\end{aligned}
$$

It can be written as

$$
\left(\begin{array}{l}
I_{1}^{\prime} \\
I_{2}^{\prime}
\end{array}\right)=\left(\begin{array}{l}
\beta_{1} S_{1} I_{2} \\
\beta_{2} S_{2} I_{2}
\end{array}\right)-\left(\begin{array}{c}
\left(\sigma_{1}+\alpha_{1}+d_{1}\right) I_{1} \\
\left(\alpha_{2}+d_{2}\right) I_{2}
\end{array}\right)=\mathcal{F}-\mathcal{V}
$$

Following the recipe from [10] we have

$$
F=\left(\begin{array}{ll}
0 & \beta_{1} S_{1}^{0} \\
0 & \beta_{2} S_{2}^{0}
\end{array}\right), \quad V=\left(\begin{array}{cc}
\sigma_{1}+\alpha_{1}+d_{1} & 0 \\
0 & \alpha_{2}+d_{2}
\end{array}\right)
$$

$F V^{-1}$ is the next generation matrix and $R_{0}$ is the spectral of $F V^{-1}$. We call $R_{0}$ as the basic reproduction number

$$
R_{0}=\frac{\beta_{2} S_{2}^{0}}{\alpha_{2}+d_{2}}
$$

\subsection{Dynamic Analysis on Disease-Free Equilibrium}

Theorem 1. If $R_{0}<1$, the disease-free equilibrium $E^{0}$ of the model is locally asymptotic stability, or else, $E^{0}$ is instability.

Proof:

The disease-free equilibrium Jacobian matrix of model (1) is 


$$
\mathcal{J}=\left(\begin{array}{cccc}
-d_{1} & \sigma_{1} & 0 & -\beta_{1} S_{1}^{0} \\
0 & -\left(\sigma_{1}+\alpha_{1}+d_{1}\right) & 0 & \beta_{1} S_{1}^{0} \\
0 & 0 & -d_{2} & -v \beta_{2} S_{2}^{0} \\
0 & 0 & 0 & \beta_{2} S_{2}^{0}-\alpha_{2}-d_{2}
\end{array}\right)
$$

The eigenvalues of $\mathcal{J}$ can be obtained from the above equation as follows:

$$
\begin{array}{ll}
\lambda_{1}=-d_{1} & \lambda_{2}=-\left(\sigma_{1}+\alpha_{1}+d_{1}\right) \\
\lambda_{3}=-d_{2} & \lambda_{4}=\beta_{2} S_{2}^{0}-\alpha_{2}-d_{2}
\end{array}
$$

when $R_{0}<1$, we can know $\lambda_{4}<0$, then every eigenvalue of the model has the negative real parts, $E^{0}$ is locally asymptotic stability. Otherwise, $E^{0}$ is unstable and there will be exist diseases case.

Theorem 2. If $R_{0}<1$, then the disease-free equilibrium $E^{0}$ is globally asymptotic stability.

Proof:

The Jacobian matrix of model (2) on the disease-free equilibrium $E^{0}$ is

$$
\mathcal{M}=\left(\begin{array}{cc}
-\left(\sigma_{1}+\alpha_{1}+d_{1}\right) & \beta_{1} S_{1}^{0} \\
0 & \beta_{2} S_{2}^{0}-\alpha_{2}-d_{2}
\end{array}\right)
$$

It is easy to see that when $R_{0}<1$, there is $\rho(\mathcal{M})<0$, where

$$
\rho(\mathcal{M})=\max \{\operatorname{Re} \lambda: \lambda \text { is an eigenvalue of } \mathcal{M}\}
$$

Since the invariant set of the model (1) is $\Omega$, choose positive and small enough $\varepsilon_{1}, \varepsilon_{2}$, and $t_{i}>0$, such for all $t>t_{i}$, there is

$$
S_{1} \leq \frac{b_{1}}{d_{1}}<S_{1}^{0}+\varepsilon_{1}, \quad S_{2} \leq \frac{b_{2}}{d_{2}}<S_{2}^{0}+\varepsilon_{2}
$$

Consider the following auxiliary model

$$
\begin{aligned}
& \tilde{I}_{1}^{\prime}=\beta_{1}\left(S_{1}^{0}+\varepsilon_{1}\right) \tilde{I}_{2}-\sigma_{1} \tilde{I}_{1}-\alpha_{1} \tilde{I}_{1}-d_{1} \tilde{I}_{1} \\
& \tilde{I}_{2}^{\prime}=\beta_{2}\left(S_{2}^{0}+\varepsilon_{2}\right) \tilde{I}_{2}-\alpha_{2} \tilde{I}_{2}-d_{2} \tilde{I}_{2}
\end{aligned}
$$

Similarly, the Jacobian matrix of model (3) on the disease-free equilibrium $E^{0}$ is

$$
\begin{aligned}
\tilde{\mathcal{M}} & =\left(\begin{array}{cc}
-\left(\sigma_{1}+\alpha_{1}+d_{1}\right) & \beta_{1} S_{1}^{0}+\varepsilon_{1} \beta_{1} \\
0 & \beta_{2} S_{2}^{0}-\alpha_{2}-d_{2}+\varepsilon_{2} \beta_{2}
\end{array}\right) \\
& =\mathcal{M}+\varepsilon_{1}\left(\begin{array}{cc}
0 & \beta_{1} \\
0 & 0
\end{array}\right)+\varepsilon_{2}\left(\begin{array}{cc}
0 & 0 \\
0 & \beta_{2}
\end{array}\right)
\end{aligned}
$$

Since $\varepsilon_{1}, \varepsilon_{2}>0$ are infinitely close to zero, $\rho(\tilde{\mathcal{M}})$ is continuous, on account of $\rho(\mathcal{M})<0$, It's easy to derive $\rho(\tilde{\mathcal{M}})<0$ for positive and small enough $\varepsilon_{1}, \varepsilon_{2}$. By the comparison principle [19], it can be concluded that $I_{1}(t) \rightarrow 0$ and $I_{2}(t) \rightarrow 0$, as $t \rightarrow \infty$. By the theory of [20], which defined that $\dot{x}=f(t, x)$ is called asymptotically autonomous-with limit equation $\dot{y}=g(y)$ if $f(t, x) \sim g(x), t \rightarrow \infty$, locally uniformly in $x \in R^{n}$.

Thus, there exist $S_{1}(t) \rightarrow S_{1}^{0}$ and $S_{2}(t) \rightarrow S_{2}^{0}$ as $t \rightarrow \infty$. Accordingly, when $R_{0}<1$, the disease-free equilibrium of the model $E^{0}$ is global asymptot- 
ic stability.

\subsection{Dynamic Analysis on Endemic Equilibrium}

We have calculated the endemic equilibrium $E^{*}$ in the above article. Next, the global exponentially stable of $E^{*}$ will be shown.

Theorem 3. If $R_{0}>1$, then the endemic equilibrium $E^{*}$ of the model is globally asymptotic stability.

Proof:

On the endemic equilibrium $E^{*}$, we derive the model (1) satisfied the follow equation:

$$
\begin{aligned}
& 0=b_{1}+\sigma_{1} I_{1}^{*}-\beta_{1} S_{1}^{*} I_{2}^{*}-d_{1} S_{1}^{*} \\
& 0=\beta_{1} S_{1}^{*} I_{2}^{*}-\sigma_{1} I_{1}^{*}-\alpha_{1} I_{1}^{*}-d_{1} I_{1}^{*} \\
& 0=b_{2}-\beta_{2} S_{2}^{*} I_{2}^{*}-d_{2} S_{2}^{*} \\
& 0=\beta_{2} S_{2}^{*} I_{2}^{*}-\alpha_{2} I_{2}^{*}-d_{2} I_{2}^{*}
\end{aligned}
$$

Thus, the model (1) can be converted into

$$
\begin{aligned}
& S_{1}^{\prime}=\sigma_{1}\left(I_{1}-I_{1}^{*}\right)-\beta_{1}\left(S_{1} I_{2}-S_{1}^{*} I_{2}^{*}\right)-d_{1}\left(S_{1}-S_{1}^{*}\right) \\
& I_{1}^{\prime}=\beta_{1}\left(S_{1} I_{2}-S_{1}^{*} I_{2}^{*}\right)-\sigma_{1}\left(I_{1}-I_{1}^{*}\right)-\alpha_{1}\left(I_{1}-I_{1}^{*}\right)-d_{1}\left(I_{1}-I_{1}^{*}\right) \\
& S_{2}^{\prime}=-\beta_{2}\left(S_{2} I_{2}-S_{2}^{*} I_{2}^{*}\right)-d_{2}\left(S_{2}-S_{2}^{*}\right) \\
& I_{2}^{\prime}=\beta_{2}\left(S_{2} I_{2}-S_{2}^{*} I_{2}^{*}\right)-\alpha_{2}\left(I_{2}-I_{2}^{*}\right)-d_{2}\left(I_{2}-I_{2}^{*}\right)
\end{aligned}
$$

Define

$$
\begin{array}{ll}
X_{1}=\frac{S_{1}}{S_{1}^{*}}-1, & X_{2}=\frac{I_{1}}{I_{1}^{*}}-1 \\
X_{3}=\frac{S_{2}}{S_{2}^{*}}-1, & X_{4}=\frac{I_{2}}{I_{2}^{*}}-1
\end{array}
$$

It is easy to get

$$
X_{1}^{\prime}=\frac{1}{S_{1}^{*}} S_{1}^{\prime}, \quad X_{2}^{\prime}=\frac{1}{I_{1}^{*}} I_{1}^{\prime}, \quad X_{3}^{\prime}=\frac{1}{S_{2}^{*}} S_{2}^{\prime}, \quad X_{4}^{\prime}=\frac{1}{I_{2}^{*}} I_{2}^{\prime}
$$

The corresponding differential equation is

$$
\begin{aligned}
& X_{1}^{\prime}=\sigma_{1} \frac{I_{1}^{*}}{S_{1}^{*}} X_{2}-\beta_{1} I_{2}^{*}\left(X_{1} X_{4}+X_{1}+X_{4}\right)-d_{1} X_{1} \\
& X_{2}^{\prime}=\beta_{1} \frac{S_{1}^{*} I_{2}^{*}}{I_{1}^{*}}\left(X_{1} X_{4}+X_{1}+X_{4}\right)-\left(\sigma_{1}+\alpha_{1}+d_{1}\right) X_{2} \\
& X_{3}^{\prime}=-d_{2} X_{3}-\beta_{2} I_{2}^{*}\left(X_{3} X_{4}+X_{3}+X_{4}\right) \\
& X_{4}^{\prime}=\beta_{2} S_{2}^{*}\left(X_{3} X_{4}+X_{3}+X_{4}\right)-\left(\alpha_{2}+d_{2}\right) X_{4}
\end{aligned}
$$

We construct the Lyapunov function as

$$
L=\frac{X_{3}^{2}}{2 \beta_{2} I_{2}^{*}}+\frac{X_{4}-\ln \left(1+X_{4}\right)}{\beta_{2} S_{2}^{*}}
$$

Therefore, the derivative of $L$ is as follow 


$$
\begin{aligned}
L^{\prime}= & \frac{X_{3}}{\beta_{2} I_{2}^{*}} X_{3}^{\prime}+\frac{1}{\beta_{2} S_{2}^{*}}\left(1-\frac{1}{1+X_{4}}\right) X_{4}^{\prime} \\
= & -\frac{X_{3}^{2} d_{2}}{\beta_{2} I_{2}^{*}}-X_{3}\left(X_{3} X_{4}+X_{3}+X_{4}\right) \\
& +\frac{X_{4}}{1+X_{4}}\left[\left(X_{3} X_{4}+X_{3}+X_{4}\right)-\frac{X_{4}}{\beta_{2} S_{2}^{*}}\left(\alpha_{2}+d_{2}\right)\right] \\
= & -X_{3}^{2}\left(\frac{d_{2}}{\beta_{2} I_{2}^{*}}+X_{4}+1\right)+\frac{X_{4}^{2}}{1+X_{4}}\left(1-\frac{\alpha_{2}+d_{2}}{\beta_{2} S_{2}^{*}}\right) \\
= & -X_{3}^{2}\left(\frac{d_{2}}{\beta_{2} I_{2}^{*}}+\frac{I_{2}}{I_{2}^{*}}\right) \leq 0
\end{aligned}
$$

It is easy to see, $L^{\prime} \leq 0$ for all $X_{3}$. In addition, if and only if $X_{3}=0$, $L^{\prime}=0$. That is to say, $L^{\prime}=0$ if and only if $S_{2}=S_{2}^{*}, I_{2}=I_{2}^{*}, S_{1}=S_{1}^{*}, I_{1}=I_{1}^{*}$. Thus, the endemic equilibrium $E^{*}$ of the model is globally asymptotic stability in the interior of $\Omega$.

\section{Numerical Simulation}

In this section, we discuss two situations in which animal population size is larger or smaller than population size. For the purpose of proving the universality of the model, we analyze the global stability by establishing the simulation data including animals and setting the parameters and initial values of the population size. After that, we give the sensitive analysis to see what is the most important factor to infect the existence or extinction of disease.

\subsection{Numerical Results}

Because it is not specific to infectious diseases, we use dimensionless parameter to conduct the numerical experiments. All the parameters values and initial values are displayed in Table 1, when people size is larger than animal size.

Since the transmission rate of animal to animal is bigger than animal to human and disease mortality rate is bigger than nature mortality rate, for $R_{0}<1$, we set $\beta_{2}=1 \times 10^{-3}$ and $\alpha_{2}=0.12$, this moment $R_{0} \approx 0.952$ the corresponding results are shown in Figure $2(\mathrm{a})$. For $R_{0}>1$, we set $\beta_{2}=1.2 \times 10^{-3}$ and $\alpha_{2}=0.16$, this moment $R_{0} \approx 1.042$ the corresponding results will be displayed in Figure 2(b).

We can conclude from Figure 2(a) that when $R_{0}<1$, the number of infected animal individuals is stable at 0 , that is to say the disease-free equilibrium $E^{0}$ is globally stable. Otherwise, from Figure $2(\mathrm{~b})$ when $R_{0}>1$, the result shows that the quantity of infected animal individuals is finally stable around 10.9, in the meantime, we calculate $I_{2}^{*}=10.86957$, it illustrates that the endemic equilibrium $E^{*}$ is globally stable when $R_{0}>1$.

Next, we analysis the situation of the people size is smaller than animal size. Values of parameters and initial values are shown in Table 2.

For $R_{0}<1$, we set $\beta_{2}=8 \times 10^{-5}$ and $\alpha_{2}=0.8$, now we calculate $R_{0} \approx 0.833$ 
Table 1. Values of parameters and initial values when people size is larger than animal size.

\begin{tabular}{ccccc}
\hline \multirow{4}{*}{ parameters } & name & value & name & value \\
\cline { 2 - 5 } & $b_{1}$ & 200 & $b_{2}$ & 120 \\
& $\sigma_{1}$ & 0.6 & $d_{2}$ & 0.3 \\
& $\beta_{1}$ & $3 \times 10^{-4}$ & $\beta_{2}$ & vary \\
& $d_{1}$ & 0.06 & $\alpha_{2}$ & vary \\
initial values & $\alpha_{1}$ & 0.2 & & \\
& name & value & name & value \\
& $S_{1}(0)$ & 2000 & $S_{2}(0)$ & 500 \\
\hline
\end{tabular}

Table 2. Values of parameters and initial values when people size is smaller than animal size.

\begin{tabular}{ccccc}
\hline & name & value & name & value \\
\cline { 2 - 5 } parameters & $b_{1}$ & 100 & $b_{2}$ & 5000 \\
& $\sigma_{1}$ & 0.4 & $d_{2}$ & 0.4 \\
& $\beta_{1}$ & $4 \times 10^{-4}$ & $\beta_{2}$ & vary \\
& $d_{1}$ & 0.08 & $\alpha_{2}$ & vary \\
initial values & $\alpha_{1}$ & 0.3 & & \\
& name & value & name & value \\
& $S_{1}(0)$ & 1000 & $S_{2}(0)$ & 10000 \\
& $I_{1}(0)$ & 20 & $I_{2}(0)$ & 200 \\
\hline
\end{tabular}
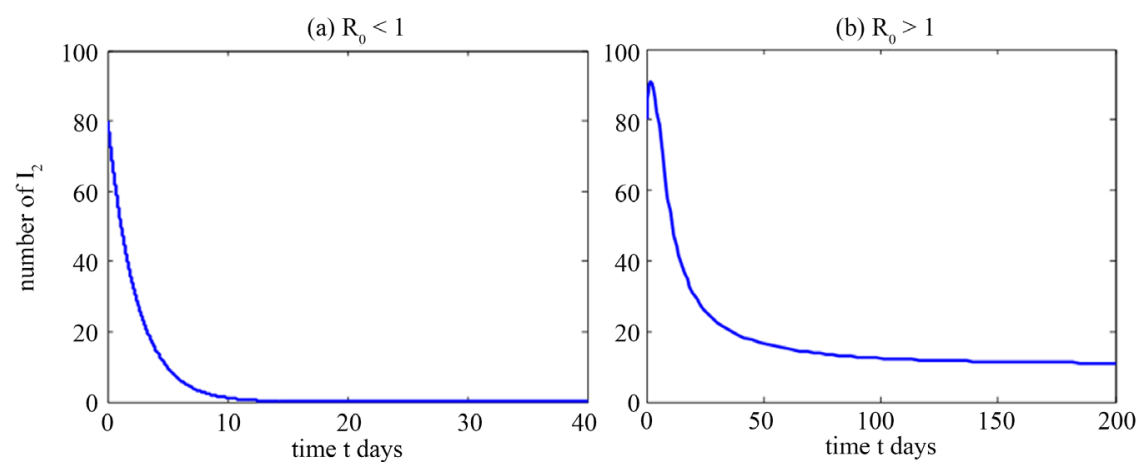

Figure 2. Numerical results of the infected animal individuals when people size is larger than animal size.

and the corresponding results are shown in Figure 3(a). For $R_{0}>1, \beta_{2}=1 \times 10^{-4}$ and $\alpha_{2}=0.7$, now $R_{0} \approx 1.136$ and the corresponding results are shown in Figure 3(b).

Similarly, Figure 3(a) we know when $R_{0}<1$, the disease-free equilibrium $E^{0}$ is globally stable. When $R_{0}>1$, the number of infected animal individuals is stable around 545.5, and we calculate $I_{2}^{*}=545.45455$, it illustrates that the endemic equilibrium $E^{*}$ is globally stable when $R_{0}>1$. 
(a) $R_{0}<1$

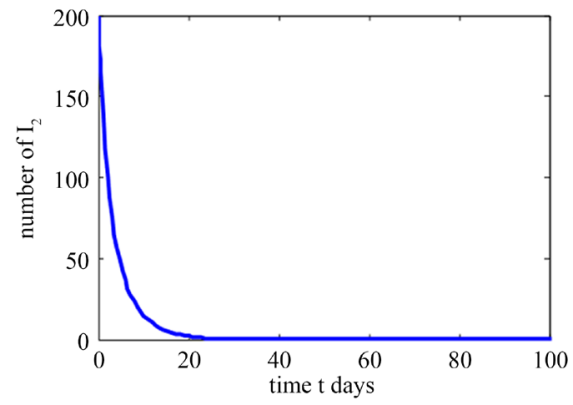

(b) $\mathrm{R}_{0}>1$

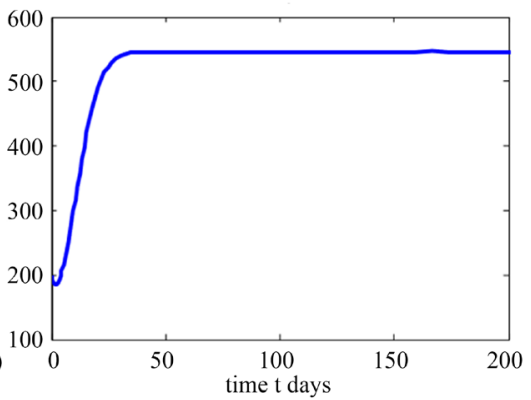

Figure 3. Numerical results of the infected animal individuals when people size is smaller than animal size.

\subsection{Sensitive Analysis}

With the view of investigating the role of each parameter on $R_{0}$, we use the following formula:

$$
\operatorname{SI}(\rho)=\frac{\rho}{R_{0}} \frac{\partial R_{0}}{\partial \rho}
$$

where $\rho$ is the parameters of $R_{0}$, and $\operatorname{SI}(\rho)$ shows the sensitivity of $R_{0}$ to $\rho$. The greater the absolute value of $\operatorname{SI}(\rho)$, the greater the effect of $\rho$ on $R_{0}$. It is easy to see $\operatorname{SI}\left(\beta_{2}\right)$ and $\operatorname{SI}\left(b_{2}\right)$ are equal to $1, \operatorname{SI}\left(d_{2}\right)$ and $\operatorname{SI}\left(\alpha_{2}\right)$ are as follows:

$$
\operatorname{SI}\left(d_{2}\right)=-\frac{2 d_{2}+\alpha_{2}}{d_{2}+\alpha_{2}}, \operatorname{SI}\left(\alpha_{2}\right)=-\frac{\alpha_{2}}{d_{2}+\alpha_{2}}
$$

Obviously, $\operatorname{SI}\left(d_{2}\right)<-1,-1<\operatorname{SI}\left(\alpha_{2}\right)<0$. It follows that increase the mortality of animal can promoting the extinction of disease. However, massive killing of animals is not advocated for those animals like dogs, sheep. So we can find ways to reduce the infection rate and birth rate.

\section{Conclusion}

In this article, we construct a general model of zoonoses transmitted from animals to humans. By dynamic analysis we can know that when $R_{0}<1$, the disease-free equilibrium $E^{0}$ is globally stable. When $R_{0}>1, E^{*}$ is globally stable. Theory tells us that infectious diseases will not unrestrictedly grow, but if not take action to solve the epidemic of infectious diseases, it will exist all the time and then continuously make harm to human society. We can take measures to control the birth rate of animals to reduce the incidence of infectious diseases.

\section{Conflicts of Interest}

The author declares no conflicts of interest regarding the publication of this paper.

\section{References}

[1] Zhang, J., Jin, Z., Sun, G.Q., Zhou, T. and Ruan, S. (2011) Analysis of Rabies in 
China: Transmission Dynamics and Control. PLOS One, 6, e20891.

https://doi.org/10.1371/journal.pone.0020891

[2] Keeling, M.J., Woolhouse, M.E.J., May, R.M., Davies, G. and Grenfell, B.T. (2003) Modelling Vaccination Strategies against Foot-and-Mouth Disease. Nature, 421, 136-142. https://doi.org/10.1038/nature01343

[3] Brauer, F. and Castillo-Chavez, C. (2001) Mathematical Models in Population Biology and Epidemiology. In: Tests in Applied Mathematics, Volume 40, Springer, New York.

[4] Leung, K.M. (2009) Development of Human Monoclonal Antibodies against Infectious Disease: SARS-Associated Coronavirus and Avian Influenza. Dissertation $A b$ stracts International, No. 1, 181.

[5] Nagao, Y. and Koelle, K. (2008) Decreases in Dengue Transmission May Act to Increase the Incidence of Dengue Hemorrhagic Fever. PNAS, 105, 2238-2243.

http://www.pnas.org/cgi/doi/10.1073/pnas.0709029105 https://doi.org/10.1073/pnas.0709029105

[6] Knobel, D.L., Cleaveland, S., Coleman, P.G., Fevre, E.M., et al. (2005) Re-Evaluating the Burden of Rabies in Africa and Asia. Bull WHO, 83, 360-368.

[7] Pappas, G., Papadimitriou, P., Akritidis, N., Christou, L. and Tsianos, E.V. (2006) The New Global Map of Human Brucellosis. The Lancet Infectious Diseases, 6, 91-99. http://linkinghub.elsevier.com/retrieve/pii/S1473309906703826 https://doi.org/10.1016/S1473-3099(06)70382-6

[8] Bremen, J.G. (2001) The Ears of the Hippopotamus: Manifestations, Determinants, and Estimates of the Malaria Burden. American Journal of Tropical Medicine and Hygiene, 64, 1-11. https://doi.org/10.4269/ajtmh.2001.64.1

[9] Sachs, J. and Malaney, P. (2002) The Economic and Social Burden of Malaria. Nature (London), 415, 680-685. https://doi.org/10.1038/415680a

[10] Kermack, W.O. and Mckendrick, A.G. (1927) A Contribution to the Mathematical Theory of Epidemics. Proceedings of the Royal Society A-Mathematical Physical and Engineering Sciences, 115, 700-721. https://www.jstor.org/stable/94815 https://doi.org/10.1098/rspa.1927.0118

[11] Kermack, W.O. and Mckendrick, A.G. (1932) Contributions to the Mathematical Theory of Epidemics. Proceedings of the Royal Society A-Mathematical Physical and Engineering Sciences, 138, 55-83. https://doi.org/10.1098/rspa.1932.0171

[12] Driessche, D.P. and Watmough, J. (2002) Reproduction Numbers and Sub-Threshold Endemic Equilibria for Compartmental Models of Disease Transmission. Mathematical Biosciences, 180, 29-48. https://doi.org/10.1016/S0025-5564(02)00108-6

[13] Yang, H.M. (2000) Malaria Transmission Model for Different Levels of Acquired Immunity and Temperature-Dependent Parameters (Vectors). Revista de Saudepublica, 34, 223-231. https://doi.org/10.1590/S0034-89102000000300003

[14] Zinsstag, J., Roth, F., Orkhon, D. Chimed-Ochir, G., Nansalmaa, M., Kolar, J., et al. (2005) A Model of Animal-Human Brucellosis Transmission in Mongolia. Preventive Veterinary Medicine, 69, 77-95.

http://linkinghub.elsevier.com/retrieve/pii/S0167587705000577 https://doi.org/10.1016/j.prevetmed.2005.01.017

[15] Elderd, B.D. and Heitman, J. (2013) Developing Models of Disease Transmission: Insights from Ecological Studies of Insects and Their Baculoviruses. PLOS Pathogens, 9, e1003372. http://dx.plos.org/10.1371/journal.ppat.1003372 https://doi.org/10.1371/journal.ppat.1003372 
[16] Herbert, H.W. (1978) An Immunization Model for a Heterogeneous Population. Theoretical Population Biology, 14, 338-349.

http://linkinghub.elsevier.com/retrieve/pii/0040580978900114

[17] Hou, Q., Sun, X.D., Wang, Y.M., Huang, B.X. and Jin, Z. (2014) Global Properties of a General Dynamic Model for Animal Diseases: A Case Study of Brucellosis and Tuberculosis Transmission. Journal of Mathematical Analysis and Applications, 414, 424-433. https://doi.org/10.1016/j.jmaa.2013.11.016

[18] Li, M., Jin, Z., Sun, G. and Zhang, J. (2017) Modeling Direct and Indirect Disease Transmission Using Multi-Group Model. Journal of Mathematical Analysis and Applications, 446, 1292-1309. https://doi.org/10.1016/j.jmaa.2016.09.043

[19] Smith, H.L. and Waltman, P. (1995) The Theory of the Chemostat. Cambridge University Press, Cambridge. https://doi.org/10.1017/CBO9780511530043

[20] Thieme, H.R. (1992) Convergence Results and a Poincare-Bendixson Trichotomy for Asymptotically Automous Differential Equations. Journal of Mathematical Biology, 30, 755-763. https://doi.org/10.1007/BF00173267 